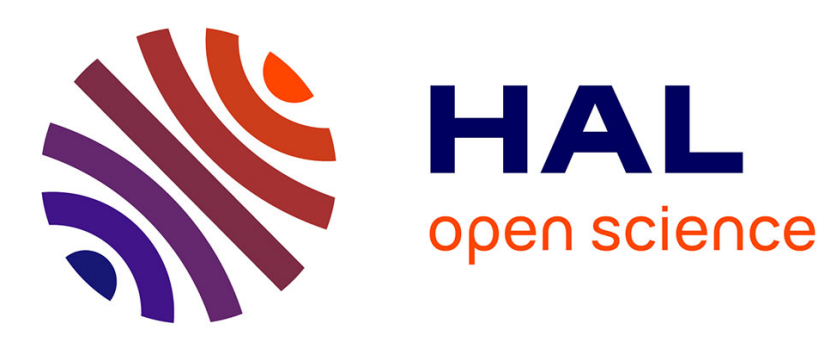

\title{
Macroscopic magnetic anisotropy in spin glasses : transverse susceptibility and zero field NMR enhancement
}

H. Alloul, F. Hippert

\section{- To cite this version:}

H. Alloul, F. Hippert. Macroscopic magnetic anisotropy in spin glasses: transverse susceptibility and zero field NMR enhancement. Journal de Physique Lettres, 1980, 41 (8), pp.201-204. 10.1051/jphyslet:01980004108020100 . jpa-00231759

\section{HAL Id: jpa-00231759 https://hal.science/jpa-00231759}

Submitted on 1 Jan 1980

HAL is a multi-disciplinary open access archive for the deposit and dissemination of scientific research documents, whether they are published or not. The documents may come from teaching and research institutions in France or abroad, or from public or private research centers.
L'archive ouverte pluridisciplinaire HAL, est destinée au dépôt et à la diffusion de documents scientifiques de niveau recherche, publiés ou non, émanant des établissements d'enseignement et de recherche français ou étrangers, des laboratoires publics ou privés. 


\title{
Macroscopic magnetic anisotropy in spin glasses : transverse susceptibility and zero field NMR enhancement
}

\author{
H. Alloul and F. Hippert \\ Laboratoire de Physique des Solides (*), Université Paris-Sud, 91405 Orsay, France \\ (Reçu le 11 janvier 1980, accepté le 25 février 1980)
}

\begin{abstract}
Résumé. - La RMN en faible champ et la susceptibilité transverse dans l'état verre de spin de CuMn confirment que toute l'aimantation rémanente $\sigma$ participe à la rotation de l'aimantation. La magnétostatique de $\sigma$ est caractérisée par des champs d'anisotropie uniaxiale $\left(H_{\mathrm{ax}}\right)$ et unidirectionnelle $\left(H_{\mathrm{d}}\right)$ déterminés indépendamment par les deux expériences. La largeur $\Delta H$ du cycle d'hystérésis est corrélée avec $H_{\mathrm{ax}}$ bien que les renversements de $\sigma$ ne soient pas parfaitement compris. L'énergie d'anisotropie mesurée dans AuFe est supérieure de deux ordres de grandeur à celle de $\underline{\mathrm{CuMn}}$.
\end{abstract}

\begin{abstract}
Low field NMR and transverse susceptibility data in CuMn spin glass confirm that all the remanence $\sigma$ is involved in the magnetization rotation. The quasi equilibrium magnetostatics of $\sigma$ is characterized by uniaxial $\left(H_{\mathrm{ax}}\right)$ and unidirectional $\left(H_{\mathrm{d}}\right)$ anisotropy fields determined by either experiment. The width $\Delta H$ of the hysteresis cycle, when detected, is correlated with $H_{\mathrm{ax}}$, although the reversals of $\sigma$ are not fully understood. The anisotropy energy is found two orders of magnitude larger in AuFe than in CuMn.
\end{abstract}

Recently zero field NMR and ESR experiments on CuMn [1], [2] for $T \ll T_{\mathrm{g}}$, have shown that the response of the electronic magnetization of such a spin glass to a radio frequency field is strongly dependent on the remanence previously established in the sample. The NMR results have demonstrated that a spin glass cannot be considered as an assembly of independent clouds with random magnetizations $\sigma_{i}$ fixed by random anisotropy fields [3]. Although a somewhat related inhomogeneous description is probably needed to describe the magnetization process of a spin glass, the NMR and ESR data in low fields, as well as the sharp hysteresis loops [4] detected in CuMn suggest that, at least for $T \ll T_{\text {g }}$, the Mn spins respond collectively to a small external field. In this letter we present direct comparisons between NMR, transverse susceptibility $\left(\chi_{\perp}\right)$, and hysteresis cycle data taken on the same samples. It will be demonstrated that, once a remanent magnetization has been established in a sample, with a given magnetothermal history, its quasi equilibrium magnetostatic behaviour, in some sense similar to that of a ferrimagnet, is characterized by well defined anisotropy fields aligned along the cooling field, which can be deduced independently

(*) Laboratoire associé au C.N.R.S. from NMR or $\chi_{\perp}$. Evidence will be given for the existence of a correlation between these anisotropy fields and the fields at which the magnetization jumps occur.

Let us briefly recall that for a system of spins rigidly bound together, with a total magnetization $\sigma / / \mathrm{z}$, a field $H_{1}$ applied in direction $\mathbf{y}$ induces a collective rotation of the spins which is limited by anisotropy forces. In the presence of a field $\mathbf{H}_{0} / / \sigma$, and if the anisotropy energy is written $E=K \sin ^{2} \psi$ with $\psi=(\boldsymbol{\sigma}, \mathbf{z})$, the rotation of $\boldsymbol{\sigma}$ corresponds to a transverse susceptibility

$$
\chi_{\sigma}=\sigma\left(H_{0}+H_{\mathrm{A}}\right)^{-1}
$$

for $H_{1} \ll H_{\mathrm{A}}$, where $H_{\mathrm{A}}=2 \mathrm{~K} / \sigma$ is the anisotropy field. In a typical low field NMR experiment [1] $H_{1}$ is a r.f. field and rotation of $\sigma$ at frequency $\omega$ induces an additional r.f. field at the nuclear site which is enhanced with respect to $H_{1}$ by a factor :

$$
\eta=\alpha H_{\mathrm{L}}\left(H_{0}+H_{\mathrm{A}}\right)^{-1},
$$

where $H_{\mathrm{L}}$ is the hyperfine field on the nucleus. In a ferro or ferrimagnet $\alpha=1$, while in a spin glass $\eta$ is an average over the random angular orientation of the local magnetizations, which corresponds to $\alpha \sim 0.9$ [1]. Previous NMR data suggest that $H_{\mathrm{A}}$ is unique and 
that most Mn spins are involved in this rotation. In order to check this point quantitatively we have measured $\chi_{\perp}$ and $\eta$ on the same CuMn $1.35 \%$ sample. The initial bulk ingot has been laminated down to $30 \mu$ thickness and then annealed at $500{ }^{\circ} \mathrm{C}$ in vacuum for about one hour. A sample with about 30 pieces taken from this foil and separated by mylar sheets was then assembled. After zero field cooling down to $1.4 \mathrm{~K}$, the isotropic ac susceptibility of this sample $\chi_{\text {iso }}=(2.38 \pm 0.04) \cdot 10^{-5} \mathrm{emu} / \mathrm{G}$ measured in direction $\mathbf{y}$ (at a frequency of $70 \mathrm{~Hz}$ ) was found independent within experimental accuracy of an external field ( $\lesssim 2 \mathrm{kG}$ ) applied either along $\mathbf{z}$ or $\mathbf{y}$ (Fig. 1). Such a field is indeed known to develop a negligible isothermal remanent magnetization in these experimental conditions [5]. In the experimental set up the field in direction $\mathrm{z}$ could only be applied below $4.2 \mathrm{~K}$ and could not exceed $2.6 \mathrm{kG}$. Therefore a partial remanence along $\mathrm{z}$,

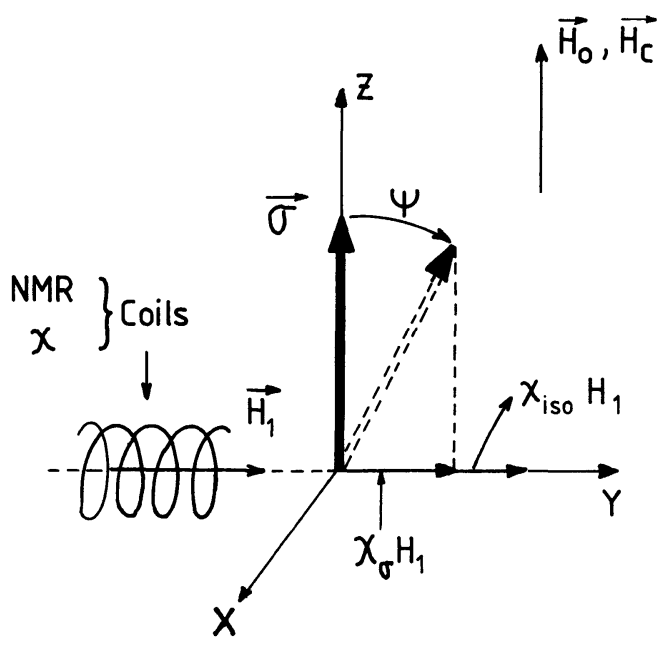

Fig. 1. - Geometry of the NMR and transverse susceptibility experiments.

$\sigma=15.8 \times 10^{-3} \mathrm{emu}$ [6] has been established by cooling the sample in zero field down to $4.2 \mathrm{~K}$, then in $H_{\mathrm{c}}^{z}=2.6 \mathrm{kG}$ down to $1.7 \mathrm{~K}$. The ac susceptibility $\chi=\chi_{\sigma}+\chi_{\text {iso }}$ was then measured at $1.44 \mathrm{~K}$ in direction $\mathbf{y}$. The inverse susceptibility $\chi_{\sigma}^{-1}=\left(\chi_{\perp}-\chi_{\text {iso }}\right)^{-1}$ due to magnetization rotation is plotted in figure 2 versus $H_{0}$. For $H_{0}=0$ the measured value

$$
\chi_{\sigma}=(2 \pm 0.2) \cdot 10^{-5} \mathrm{emu} / \mathrm{G}
$$

yields $H_{\mathrm{A}}=\sigma / \chi_{\sigma}=790 \pm 80 \mathrm{G}$ assuming the applicability of eq. (1).

We have then detected the ${ }^{63} \mathrm{Cu}$ zero field NMR of the 4th nearest neighbour nuclei of the $\mathrm{Mn}$ at $\omega=10.5 \mathrm{MHz}$ in the same experimental conditions [1], and could measure the enhancement factor $\eta=12.3 \pm 2$, which yields a similar value

$$
H_{\mathrm{A}}=660 \pm 120 \mathrm{G}
$$

from eq. (2). Let us consider now the variation with $H_{0}$ of $\chi_{\sigma}^{-1}$ and $\eta^{-1}$. As has already been shown in other

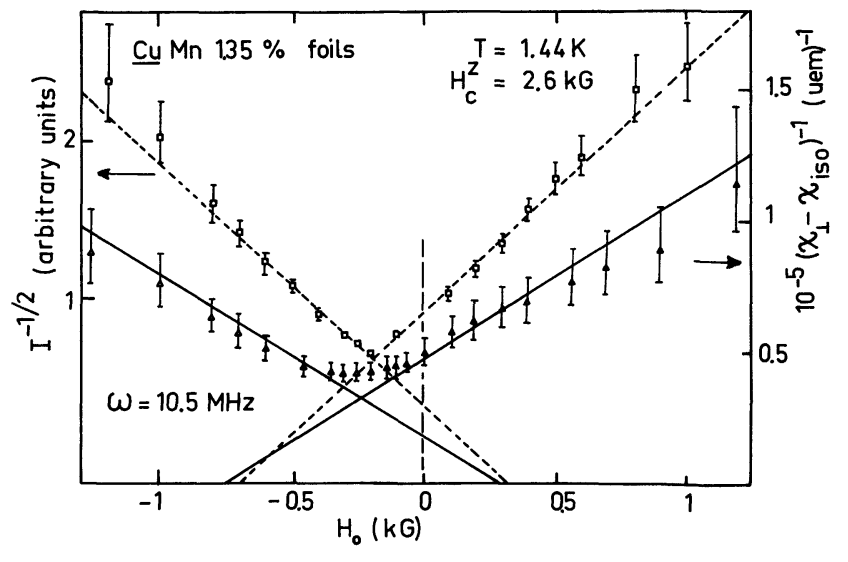

Fig. 2. - Inverse of the transverse susceptibility $\chi_{\sigma}=\chi_{\perp}-\chi_{\text {iso }}$ due to magnetization rotation and of the enhancement factor $\eta$ (proportional to the square root of the NMR signal intensity $I$ ), plotted versus $H_{0}$. For $H_{0}>0$ the data are found to agree with eqs. (1) and (2) with the values of $H_{\mathrm{A}}$ given in the text. In order to explain the results for all fields two anisotropy fields $H_{\mathrm{d}}$ and $H_{\mathrm{ax}}$ must be defined and agreement with eqs. (4) and (5) is obtained with respectively $H_{\mathrm{d}}=235 \mathrm{G}, H_{\mathrm{ax}}=515 \mathrm{G}$ (full lines) and $H_{\mathrm{d}}=190 \mathrm{G}$, $H_{\mathrm{ax}}=500 \mathrm{G}$ (dashed lines). Notice that, for the susceptibility results the slope of the full curves are obtained from the measured value of the remanent magnetization $\sigma$. The slight difference between the respective behaviour of $\chi_{\sigma}^{-1}$ and $\eta^{-1}$ for $H_{0} \sim-H_{\mathrm{d}}$ might be assigned to the fact that the reversal of $\sigma$ is gradual in these experimental conditions, for which the remanence is not saturated.

experimental conditions [1] the NMR signal in tensity $I$ is proportional to $\eta^{2}$, and the linear variation of $I^{-1 / 2}$ for $H_{0}>0$ plotted in figure 2 yields $H_{\mathrm{A}}=700 \pm 50 \mathrm{G}$ which agrees with the zero field result. Similarly, it can be seen on figure 2 that $\chi_{\sigma}^{-1}$ has a linear dependence with $H_{0}$, which fits eq. (1), the slope being given by the experimental value of $\sigma$ and $H_{\mathrm{A}}$ by the zero field value $H_{\mathrm{A}}=790 \pm 80 \mathrm{G}$. The perfect agreement between these experimental results confirms that $H_{\mathrm{A}}$ is a well defined macroscopic quantity [7] induced by the cooling field $H_{\mathrm{c}}$ and demonstrates that all the remanent magnetization is involved in the magnetization rotation process.

However agreement with the present model for $H_{0}>0$ does not allow us to decide whether $H_{\mathrm{A}}$ is indeed an uniaxial anisotropy field. This can be done by considering the data for $H_{0}<0$, as it is known that $\sigma$ can be reversed in large enough negative field. In the experimental conditions of figure 2 this reversal is gradual and only completed for $\left(H_{0}\right) \gtrsim 600 \mathrm{G}$ [8]. In such fields one should therefore expect

$$
\eta \alpha \chi_{\sigma}=|\sigma|\left(H_{\mathrm{A}}-H_{0}\right)^{-1}
$$

if $H_{\mathrm{A}}$ is a uniaxial anisotropy. The data are instead found symmetrical with respect to

$$
H_{0}=-H_{\mathrm{d}} \simeq-200 \mathrm{G} \text {. }
$$

Such a displacement field $H_{\mathrm{d}}$ has been found quite generally in the NMR [1] as well as for the magnetization curves [4], [9] and can be interpreted as a unidi- 
rectional anisotropy field [9]. Then the uniaxial anisotropy involved in $H_{\mathrm{A}}$ is only $H_{\mathrm{ax}}=H_{\mathrm{A}}-H_{\mathrm{d}}$, and the magnetostatic energy of the spin glass with remanence $\sigma$ can then be written

$$
E=-\sigma H_{0} \cos \psi-K_{\mathrm{d}} \cos \psi+K_{\mathrm{ax}} \sin ^{2} \psi,
$$

in which case $H_{\mathrm{d}}=K_{\mathrm{d}} / \sigma, H_{\mathrm{ax}}=2 K_{\mathrm{ax}} / \sigma$, and

$$
\begin{gathered}
\chi_{\sigma}=|\sigma|\left(H_{\mathrm{ax}}+\varepsilon\left(H_{0}+H_{\mathrm{d}}\right)\right)^{-1} \\
\eta=\alpha H_{\mathrm{L}}\left(H_{\mathrm{ax}}+\varepsilon\left(H_{0}+H_{\mathrm{d}}\right)\right)^{-1}
\end{gathered}
$$

with $\varepsilon=\operatorname{sgn}\left(\boldsymbol{\sigma} \cdot \mathbf{H}_{\mathrm{c}}\right)$.

Assuming that the sample behaves as an assembly of independent monodomains (at least delimited by the size of the individual foils), the jumps of $\sigma$ should take place at $H_{0}=-H_{\mathrm{d}} \pm H_{\mathrm{ax}}$ if eq. (3) is also valid at large angles. In experimental conditions for which magnetization jumps are known to occur they could be either detected directly or equivalently as the reversals of the crossed coil NMR signal [1]. In all cases (table I) the centre $-H_{\mathrm{d}}^{\prime}$ of the hysteresis cycle was found near $-H_{\mathrm{d}}$ while its width $\Delta H$ was about a factor two or three smaller than $H_{\mathrm{ax}}$. This discrepancy might be eventually solved by introducing higher order terms in $\sin ^{2 n} \psi$ in the anisotropy energy. A direct probe for such a form of the anisotropy energy might be given by torque experiments [10]. However various experimental facts point out that the reversals of $\sigma$ involve more complicated physical phenomena. Indeed in the experimental conditions of figure 2, although $H_{\mathrm{ax}}$ and $H_{\mathrm{d}}$ could be determined from NMR and $\chi_{\perp}$ data, abrupt magnetization jumps could not be detected [8]. Such complications are already apparent in the magnetization data as the up and down jumps were not found to behave similarly in the presence of a small transverse field [4]. Further explanation of the magnetization jumps certainly requires to take into account the nucleation energy of a domain wall, its propagation, etc...

However it is interesting to point out here that the relationship $H_{\mathrm{ax}} / 3 \lesssim \Delta H \lesssim H_{\mathrm{ax}} / 2$ has been found to hold for various $\mathrm{Mn}$ concentrations or remanence $\sigma$ as long as magnetization jumps can be observed (table I). For instance both $H_{\text {ax }}$ and $\Delta H$ are about two times larger for a powdered non annealed sample than for the foil sample taken from the same CuMn $1.35 \%$ bulk ingot. Therefore although a direct proportionality between $H_{\mathrm{ax}}$ and $\Delta H$ is not granted these quantities are correlated, and the variations of $\Delta H$ with magnetothermal history, metallurgy [4] or with $\mathrm{Au}$ content in $\mathrm{CuAu}_{y} \mathrm{Mn}_{x}$ [11] alloys certainly give a good estimate of the corresponding variation of $H_{\mathrm{ax}}$. In particular this recent experimental study allows us to characterize the influence of spin orbit scattering of the conduction electrons on $H_{\mathrm{ax}}$ and $H_{\mathrm{d}}$, and therefore sheds some light on the origin of these anisotropies. It has then been quite interesting to consider the case of AuFe for which no jumps in the hysteresis cycle have been detected to our knowledge. We have therefore measured the zero field transverse susceptibility $\chi_{\perp}$ of a $\mathrm{AuFe} 3.7 \%$ sample with saturated remanent magnetization at $1.3 \mathrm{~K}$. In such a sample the anisotropy of the susceptibility is much smaller than in $\mathrm{CuMn}$, as $\chi_{\text {iso }}=(1.47 \pm 0.05) \cdot 10^{-5} \mathrm{emu} / \mathrm{G}$, $\chi_{\sigma}=(1.28 \pm 0.20) \cdot 10^{-6} \mathrm{emu} / \mathrm{G}$, which with

$$
\sigma=(6.8 \pm 0.4) \cdot 10^{-3} \mathrm{emu} / \mathrm{G}
$$

taken from an extrapolation of the remanence data for a AuFe $3 \%$ sample [12], yields $H_{\mathrm{A}}=50 \pm 10 \mathrm{kG}$ from eq. (1). This large value for $H_{\mathrm{A}}$ corresponds to an anisotropy energy two orders of magnitude larger than for $\mathrm{CuMn}$ at comparable concentration. This explains the failure to detect magnetization jumps in this system and confirms the influence of spin orbit coupling either on the impurity site, or in the matrix conduction band on the anisotropy energy.

The present results have established the close connection between the magnetization and NMR data which confirms the existence of a well defined collective response to an external field in CuMn spin glass in presence of a remanence [13]. This macroscopic behaviour has been characterized by two anisotropy fields $H_{\mathrm{ax}}$ and $H_{\mathrm{d}}$ induced by the cooling field $H_{\mathrm{c}}$,

Table I. - Comparison of the anisotropy fields $H_{\mathrm{ax}}$ and $H_{\mathrm{d}}$ determined from $\mathrm{NMR}$, with the width $\Delta H$ and position - $H_{\mathrm{d}}^{\prime}$ of the hysteresis cycles. In all cases, measurements have been performed after saturation of the remanence at $1.3 \mathrm{~K}$ and $H_{\mathrm{ax}}$ and $H_{\mathrm{d}}$ have been obtained as in figure 2 from eq. (5).

(a) NMR experiments only give approximate estimates of $\Delta H$ and $H_{\mathrm{d}}^{\prime}$ as the signal jumps are somewhat rounded due to technical limitations.

(b) P. Monod and J. J. Préjean (private communication).

$\begin{array}{cccccc}\begin{array}{c}\text { Sample } \\ -\end{array} & \text { Experiment } & H_{\mathrm{d}}(\mathrm{G}) & H_{\mathrm{ax}}(\mathrm{G}) & \Delta H(\mathrm{G}) & H_{\mathrm{d}}^{\prime}(\mathrm{G}) \\ \begin{array}{c}\text { CuMn } 1.35 \% \\ \text { foils }\end{array} & \begin{array}{c}\text { NMR } \\ \text { Magnetization }\end{array} & 75 \pm 20 & 300 \pm 50 & \sim 100 & \sim 110 \\ \begin{array}{c}\text { fuMn } 4.7 \% \\ \text { foils }\end{array} & \text { NMR } & 160 \pm 25 & 345 \pm 40 & \sim 140 & \sim 20 \pm 10 \\ \begin{array}{c}\text { CuMn } 1.35 \% \\ \text { powder }\end{array} & \text { NMR } & 225 \pm 50 & 580 \pm 80 & \sim 300 & \sim 200 \\ & & & & & \end{array}$


which have familiar physical significances. It must be emphasized that $H_{\mathrm{ax}}$ and $H_{\mathrm{d}}$ characterize a quasi equilibrium state in as much as we can neglect their time dependences after the removal of $H_{\mathrm{c}}$, which could be detected in all these experiments. As illustrated in the particular case of AuFe, transverse susceptibility measurement appear to be a simple direct technique to obtain an estimate of the anisotropy field, although further experiments are required in other systems than CuMn in order to ensure the existence of well defined macroscopic anisotropy fields and hence the applicability of the collective magnetization rotation model. Finally, although the marked influence of the spin orbit scattering on $H_{\mathrm{d}}$ and $H_{\mathrm{ax}}$ has been recognized, which allows us to suggest a plausible contribution to $H_{\mathrm{ax}}$, the origin of the unidirectional anisotropy field $H_{\mathrm{d}}$ is still puzzling. Is there a small fraction of the Mn spins not involved in the collective motion, which would allow us to associate $H_{\mathrm{d}}$ with an average exchange field due to these spins, as suggested initially by Kouvel [9] ? In such a case why is $H_{\mathrm{d}}$ influenced by a modification of the spin orbit scattering of the conduction electrons? Further resonance studies might help to clarify this point and to understand the dynamic properties of the remanence as well.

Acknowledgments. - We should like to acknowledge P. Monod for many stimulating discussions and for communication of his magnetization data. We should also like to thank J. J. Préjean for his interest and for measuring $\sigma$ in the experimental conditions of figure 2 and E. Vellu and J. P. Renard for kindly helping us to perform the ac susceptibility measurements in their apparatus.

\section{References}

[1] Alloul, H., Phys. Rev. Lett. 43 (1979) 603. More details will be found in H. Alloul MMM Conference (New York) 1979 ; J. Appl. Phys. 50 (1979) 7330.

[2] MonOd, P. and Berthier, Y., ICM 79 Munich (to be published)

[3] Holtzberg, F., Tholence, J. L. and Tournier, R., in Amorphous Magnetism II edited by R. A. Levy and R. Hasegawa (Plenum Press, New York) 1977, p. 55.

[4] Monod, P. and Préjean, J. J., J. Physique Colloq. 39 (1978) C 6-910 and Monod, P., Préjean, J. J. and Tissier, B., J. Appl. Phys. 50 (1979) 7324.

[5] Careaga, A., Thesis Grenoble (1967) unpublished.

[6] This measurement of $\sigma$ has been performed by J. J. Préjean.

[7] Attempts to fit the data with an eventual distribution of $H_{\mathrm{A}}$ values point out that the half intensity width $\Delta H_{\mathrm{A}}$ of this distribution can in no case exceed $0.25 H_{\mathrm{A}}$.
[8] Monod, P. and Présean, J. J., private communication.

[9] Kouvel, J. S., J. Phys. Chem. Solids 21 (1961) 57.

[10] Iwata, T., KaI, K., Nakamichi, T. and Yamamoto, M., J. Phys. Soc. Japan 28 (1970) 582.

[11] Pré́ean, J. J., Joliclerc, M. J. and Monod, P. (to be published in J. Physique).

[12] Tholence, J. L. and Tournier, R., J. Physique Colloq. 35 (1974) C4-229.

[13] It has been found that the anisotropy fields deduced from the zero field ESR frequencies are in excellent agreement with those deduced from the NMR (or $\chi_{\perp}$ ) data. H. Alloul, F. Hippert, H. Hurdequint and P. Monod (in preparation). 